\title{
Novel Types of AC Motors and Drives for Electrical and Hybrid Vehicles
}

\author{
Andrey Brazhnikov \\ Department of Fire Safety, Siberian Federal University, Krasnoyarsk, Russia
}

Email address:

andrey-brazhnikov@mail.ru

\section{To cite this article:}

Andrey Brazhnikov. Novel Types of AC Motors and Drives for Electrical and Hybrid Vehicles. Journal of Electrical and Electronic

Engineering. Vol. 5, No. 1, 2017, pp. 13-22. doi: 10.11648/j.jeee.20170501.13

Received: January 18, 2017; Accepted: March 13, 2017; Published: March 15, 2017

\begin{abstract}
The study of AC inverter drives in the case when the number of their phases is more than four allows to find out some basic laws of these systems which does not manifest themselves when the phase number is equal to three or four. Two previously unknown laws of energy efficiency invariance are established in the field of multiphase (i.e. having the number of phases more than four) AC inverter drives. These laws ignoring leads necessarily to the decrease of the multiphase drive system energy efficiency.
\end{abstract}

Keywords: Index Terms-AC Motors Control, Energy Efficiency, Variable Speed Drives

\section{Introduction}

The study of AC inverter drives (ACID) in the case when the number $m$ of ACID phases is more than four allows to find out some basic laws of these systems which do not manifest themselves when $m$ is equal to three or four. A knowledge of these peculiar laws is of not only scientific (i.e. cognitive), but also great practical importance because these laws ignoring leads necessarily to the decrease of the ACID energy efficiency when $m \geq 5$.

By present time two previously unknown laws of spacetemporal spectral relations have been established by the authors of this paper as a result of the corresponding investigations [1-12, 14, 17-19]. These laws are true for the case when the motor winding set is symmetric and electromagnetic processes in ACID are steady-state. They are essentially the laws of energy efficiency invariance for the field of multiphase (i.e. having the number of phases more than four) $\mathrm{AC}$ inverter drives $[13,15,16]$.

\section{Law of $m$-invariance}

The first of these two laws links the ACID efficiency $N$, phase number $m \geq 5$ and relative spectra $U^{*}(c)$ and $B^{*}(n)$, where $U^{*}(c)$ is the relative spectrum of the output (phase) voltage $u(t)$ of inverter that is also the AC motor stator phase voltage, $c$ is the number of the voltage $u(t)$ harmonic (i.e. the number of a time harmonic), $B^{*}(n)$ is the relative spectrum of the function $b(g)$ which describes the space distribution of the magnetic induction created by each phase winding of the $\mathrm{AC}$ motor stator in the machine air gap within the limits of the motor pole pitch, $n$ is the number of the function $b(g)$ harmonic (i.e. the number of a space harmonic), $t$ is a time, and $g$ is the space coordinate, which is plotting on the space coordinate curved axis $O g$, that runs along entire length of the AC motor air gap ( $g \in[0 ; 360]$, electrical degrees).

The above-mentioned relative spectra differ from the corresponding absolute (real) spectra in that the amplitude $A^{*}(x)$ of some relative spectrum harmonic is equal to

$$
A^{*}(x)=A(x) / A(1),
$$

where $A(x)$ is the amplitude of the corresponding (i.e. of the same name) harmonic of the appropriate absolute (real) spectrum and $x$ is the number of a harmonic $\left(x \equiv c\right.$ for $U^{*}(c)$ and $x \equiv n$ for $\left.B^{*}(n)\right), A(1)$ is the amplitude of one or another above-mentioned absolute (real) spectrum harmonic, number of which $x=1$. It is obviously that the following equation is always fulfilled: $A^{*}(x)=1$.

The values $U^{*}(c)$ and $B^{*}(n)$ for all $c$ and $n$ are dimensionless. Therefore both their envelope lines may be constructed on the common two-dimensional subspace (plane) $k O d$ (see Figure 1), where the axis $O k$ is horizontal, the axis $O d$ is vertical, $k \equiv c$ for $U^{*}(c)$, and $k \equiv n$ for $B^{*}(n)$. 
The values $U^{*}(c)$ and $B^{*}(n)$ are plotting on the axis $O d$.

The above-mentioned law is given the title "law of ACID efficiency $N$ invariance to the ACID phase number $m$ " (or more simply, "law of $m$-invariance"). It is stated as follows: if $b(g)=$ const when $m=$ var, then the envelope line of spectrum $U^{*}(k)$ must lie not above the envelope line of spectrum $B^{*}(k)$ on the plane $k O d$ to ensure the invariability of the ACID efficiency $N$ when the ACID phase number $m$ is changing (see Figure 1, where $I$ is the area, where the law of $m$-invariance is fulfilled, and $I I$ is the area, where the law of $m$-invariance is not fulfilled). Besides, the frequency composition of the function $b(g)$ must be identical (at least) to the frequency composition of the voltage $u(t)$ or be wider than it.

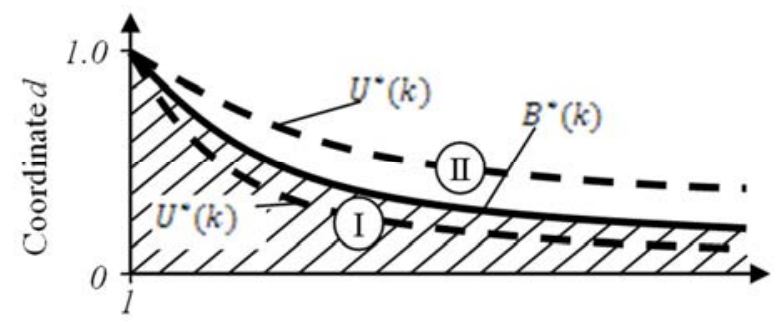

Figure 1. Illustration of the law of m-invariance.

The mathematical notation of the above mentioned law of $m$-invariance may have the following form:

$$
\begin{aligned}
& \forall m,[b(g)=\text { const }] \wedge\left[U^{*}(k) \leq B^{*}(k)\right] \Rightarrow \\
& \Rightarrow[N=\text { const }] .
\end{aligned}
$$

The identity $U^{*}(k) \equiv B^{*}(k)$ is a particular (the limiting) case of the law of m-invariance.

The fulfillment of the law of m-invariance can be achieved owing to the use of the corresponding frequency converter control algorithm (in particular, the switching algorithm of the transistors of the inverter, which is a part of a frequency converter) and the phase stator windings placement version (in particular, the number of stator slots per a phase and pole).

This law is in some contrast with widely known notion of ACID which was obtained as a result of researches only in the field of 3- and 4-phase AC drives. In particular, according to this notion the sinusoidal version of the function $b(g)$ is always considered the optimal version.

Four limiting cases, when the functions $u(t)$ and $b(g)$ have form of the sinusoid curve or right-angled meander, are presented in Table 1 for illustration of the law of $\mathrm{m}$ invariance. At this point, the sinusoidal and right-angled meander types of the functions $u(t)$ and $b(g)$ are seen as the limiting types of these functions not in terms of their spectra, but in terms of frequency converter control complexity and stator winding set intricacy. The right-angled meander type of the functions $u(t)$ corresponds to the 180-degree switching of the transistors of the inverter, which is a part of a frequency converter. The right-angled meander type of the functions $b(g)$ corresponds to the placement of every phase stator winding in two stator slots (when the AC motor design version is traditional).

Table 1. Combinations of Limiting Types of Functions $u(t)$ and $b(g)$.

\begin{tabular}{lllll}
\hline Case & Type of function $\boldsymbol{b}(\boldsymbol{g})$ & Type of function $\boldsymbol{u ( t )}$ & Behavior of $\boldsymbol{N}$ when $\boldsymbol{m}=$ var & Fulfillment or unfulfillment of law of $\boldsymbol{m}$-invariance \\
\hline 1 & Sinusoidal & Sinusoidal & $N=$ const & Fulfillment \\
2 & Sinusoidal & Right-angled meander & $N=$ var & Unfulfillment \\
3 & Right-angled meander & Sinusoidal & $N=$ const & Fulfillment \\
4 & Right-angled meander & Right-angled meander & $N=$ const & Fulfillment \\
\hline
\end{tabular}

In the case 2 (see Table 1) the ACID efficiency $N$ decreases at the increase in the phase number $m$.

The data, which are presented in Table I, was obtained by the authors as a result of their corresponding researches [1-3]. These data lends support to the validity of the law of $m$ invariance.

In the case 2 (see Table 1 ), when the function $b(g)$ is sinusoidal and the function $u(t)$ has form of right-angled meander, the transition from unfulfillment to fulfillment of the law of $m$-invariance can be achieved by the corresponding change of the frequency converter control algorithm.

\section{Law of $\boldsymbol{H}$-invariance}

The second law concerns the phase-pole controlled multiphase ACID, i.e. when the phase-pole control method (PPM) is used in ACID [4-12, 18]. This law links the ACID efficiency $N$, relative spectrum $U^{*}(c)$ relative spectrum $B^{*}(n)$ and integer-valued parameter $H$ of PPM, where $H \geq 1$ (the value $H=1$ corresponds to a traditional control mode, and the value $H>1$ corresponds to PPM).

The essence of the control according to PPM is that in this case the electrical angles between the voltages (or currents) of the nearest phases of inverter increase by a factor of some whole number $H$ without any change of the inverter voltage (or current) amplitude and frequency. During PPM application process, when the parameter $H$ changes, the effect adequate to the synchronous change of the ACID phase number and number of motor poles appears.

The above-mentioned second law is given the title "law of ACID efficiency $N$ invariance to the parameter $H$ PPM" (or more simply, "law of $H$-invariance"). It is stated as follows: if $u(t)=$ const when $H=$ var then the identity $B^{*}(n) \equiv B^{*}(H \cdot n)$ must be provided to ensure the invariability of the ACID efficiency $N$ when the parameter $H$ is changing during PPM application process. Besides, for the mentioned purpose the law of $m$-invariance must also be fulfilled to spectra $U^{*}(c)$ and $B^{*}(n)$ for all values of parameter $H$ (see Figure 2, where the line 1 is the $n$-dependence of 
$B^{*}(n)$ for $H=1$ the line 2 is the $n$-dependence of $B^{*}(H \cdot n)$ for $H=2$ and the line 3 is the $c$-dependence of $\left.U^{*}(c)\right)$.

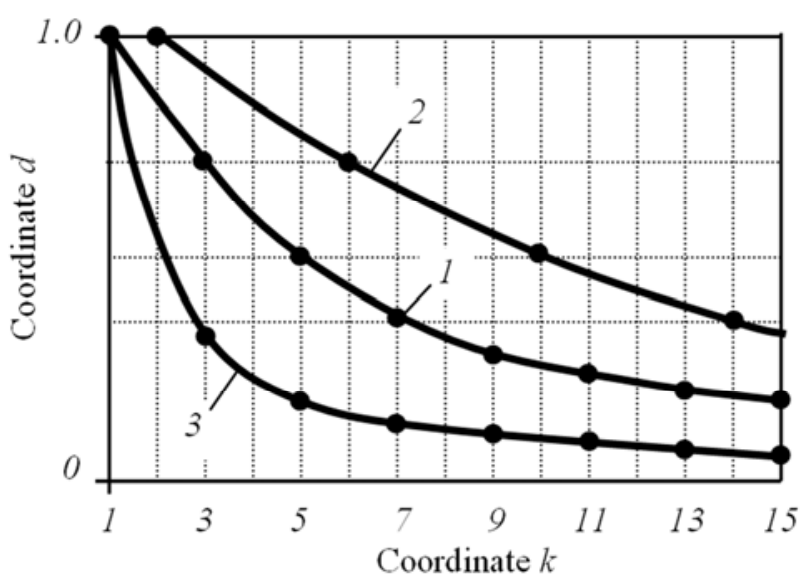

Figure 2. Illustration of the law of H-invariance.

The mathematical notation of the above mentioned law of $H$-invariance may have the following form:

$$
\begin{aligned}
\forall H,[u(t)=\mathrm{const}] \wedge\left[B^{*}(n) \equiv B\right. & *(H \cdot n)] \Rightarrow \\
& \Rightarrow[N=\text { const }] .
\end{aligned}
$$

\section{Ignoring Laws of Energy Efficiency Invariance}

If the above-mentioned laws of ACID efficiency invariance are not fulfilled, then the ACID efficiency $N$ decreases and when the ACID phase number $m$ increases more than four and when the going from some traditional control mode to PPM is being attained. For example, $m$ dependence of the ACID efficiency $N$ is presented in Fig. 3 for the case when the function $b(g)$ is sinusoidal and $t$ dependence of the voltage $u(t)$ has form of right-angled meander [1] (in this case the law of $m$-invariance is not fulfilled; see case 2 in Table 1).

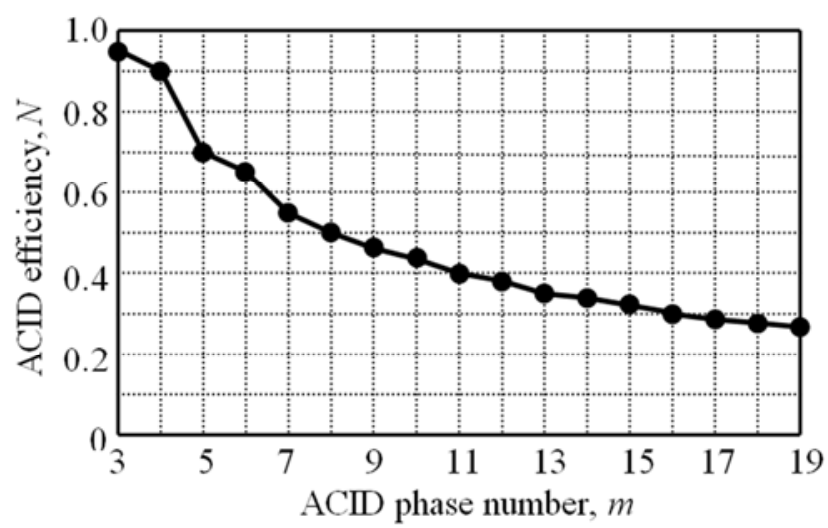

Figure 3. m-dependence of the ACID efficiency $N$ for the case when the function $b(g)$ is sinusoidal and $t$-dependence of the voltage $u(t)$ has form of right-angled meander

It follows from Figure 3 that if the law of $m$-invariance is not fulfilled, the more is the ACID phase number $m$, the less is the ACID efficiency $N$ (when $U^{*}(c)=$ const and $B^{*}(n)=$ const at $m=v a r$ ).

It should be noted the following in addition to the law of $m$-invariance. The results of the investigations carried out by the authors of this paper $[1-3,19]$ show that in the case, when the law of $m$-invariance is not fulfilled (i.e. if $U^{*}(k)>B^{*}(k)$ ), the more is value $\Delta$, the less is the ACID efficiency $N$ at the given value $m$, where

$$
\Delta=\lim _{k_{m} \rightarrow \infty} \frac{1}{k_{m}} \int_{1}^{k_{m}}\left[U^{*}(k)-B^{*}(k)\right] d k .
$$

In support of the $H$-invariance law the mechanical characteristics (i.e. $w-M$ characteristics) of some multiphase phase-pole controlled induction motor with $m \geq 6$ are presented in Figure 4 for the cases when $H=1$ (see line 1) and $H=2$ (see lines 2 and 3), where the line 2 is the mechanical characteristic if the law of $H$-invariance is fulfilled, line 3 the mechanical characteristic if the law of $\mathrm{H}$ invariance is not fulfilled, $w$ is the speed of rotation, and $M$ is the motor torque [4-12, 14, 17-19].

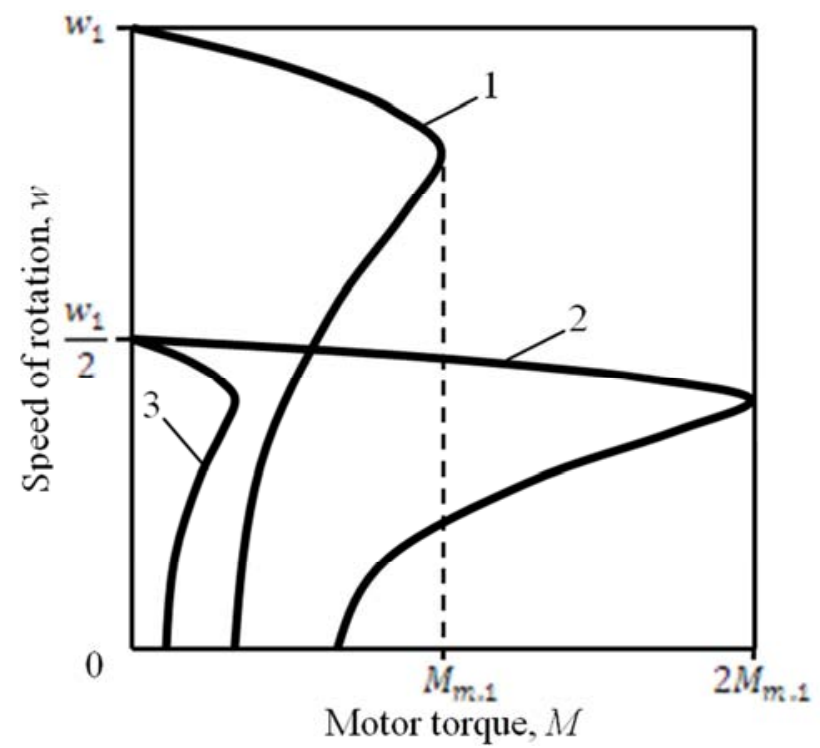

Figure 4. Mechanical characteristics of some multiphase phase-pole controlled induction motor with $m \geq 6$ for the cases when $H=1$ (see line 1) and $H=2$ (see lines 2 and 3 ).

\section{Design Implementation of Law of $\boldsymbol{H}$-invariance}

The law of $H$-invariance reduces to the following two conditions, which must be fulfilled to achieve the PPM application opportunity:

Condition 1: The space harmonics having numbers $n=p \cdot H$ must be contained in the spectrum of the function $b(g)$ describing the space distribution of the mutual inductances between motor phase windings for all parameter $H$ values which have to be realized in the given drive system.

Condition 2: When $H>1$, the amplitudes of the above mentioned space harmonics (i.e. having numbers $n=p \cdot H$ ) 
must have the values being not less than those at $H=1$.

It is obviously that the fulfillment of these conditions (i.e. the above mentioned law of $H$-invariance) may be provided only with obtaining the conductive decoupling between the AC motor stator slots, which cannot be achieved if design of the motor and its stator winding set are traditional.

Because of this it is necessary to use some peculiar designs of multiphase AC liner and non-linear motors to ensure fulfillment of the $H$-invariance law [9, 14, 20].

According to this deduction the following three basic design versions were developed by the author of this paper:

Basic design version 1 (BDV-1). The AC motor stator winding set consists of the individual rods located in stator slots and insulated from stator magnetic circuit [29] (Figures 5, 6 , and 7).

Basic design version 2 (BDV-2). The AC motor stator has toroidal-type winding set [26]. The motor rotor envelops the stator both on the outside and on the inside (when the motor is of rotating type) or from above and from below (when the motor is linear or bow-shaped).

Basic design version 3 (BDV-3). The AC motor stator core (i.e. magnetic circuit) consists of two parts magnetically separated from each other by diamagnetic shield.

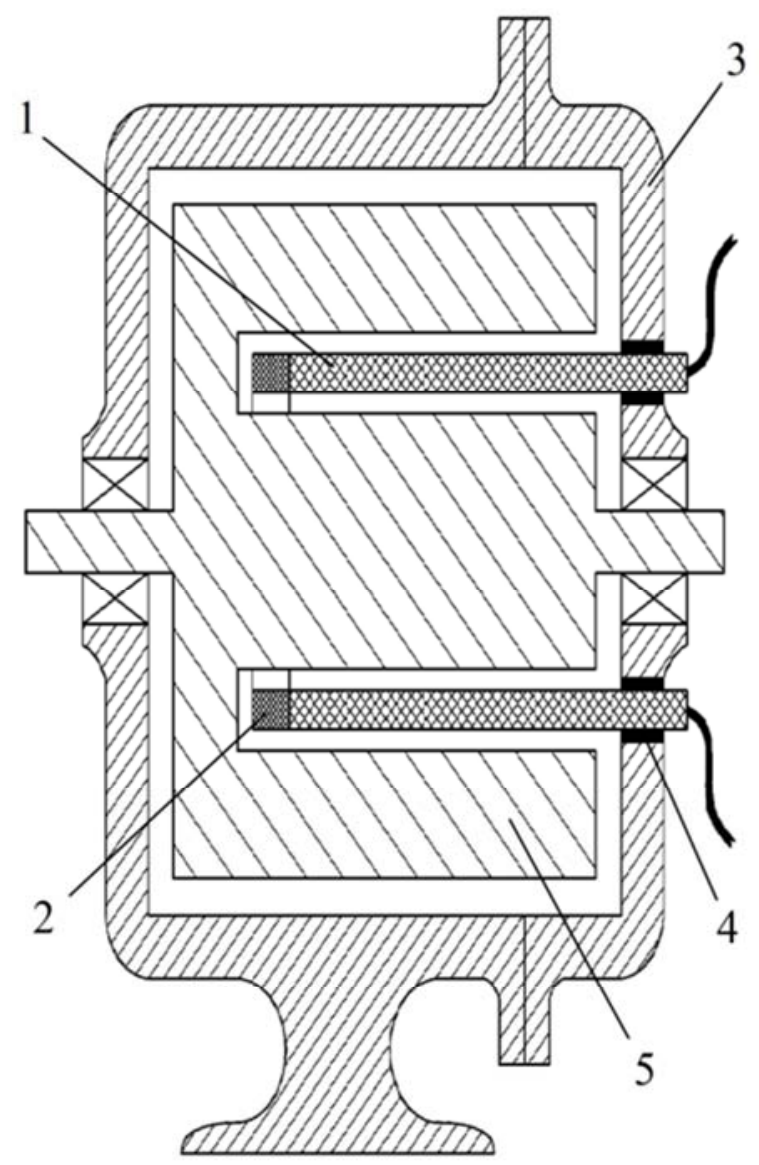

Figure 5. The section of multiphase induction motor of rotating type worked out according to the basic design version $B D V-1$, where 1 are rods of the stator winding sets (the stator has not any magnetic circuit), 2 shortcircuited ring, 3 is the motor case, 4 are insulators, and 5 is the E-shaped rotor core (magnetic circuit).

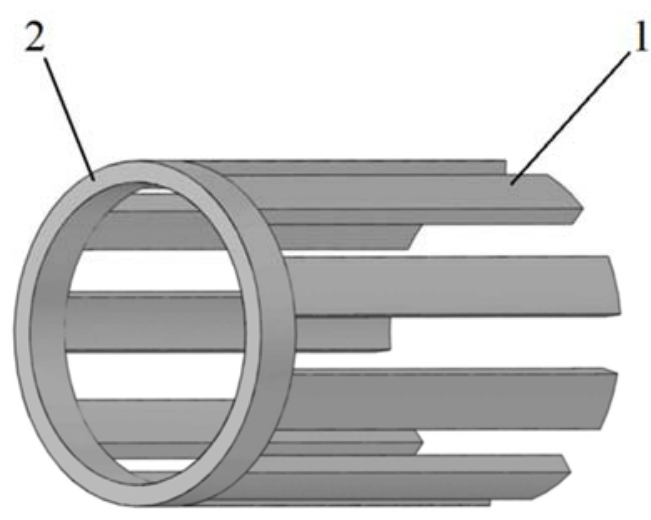

Figure 6. The stator of multiphase induction motor of rotating type worked out according to the basic design version $B D V-1$, where 1 are rods of the stator winding sets (the stator has not any magnetic circuit), and 2 is shortcircuited ring.

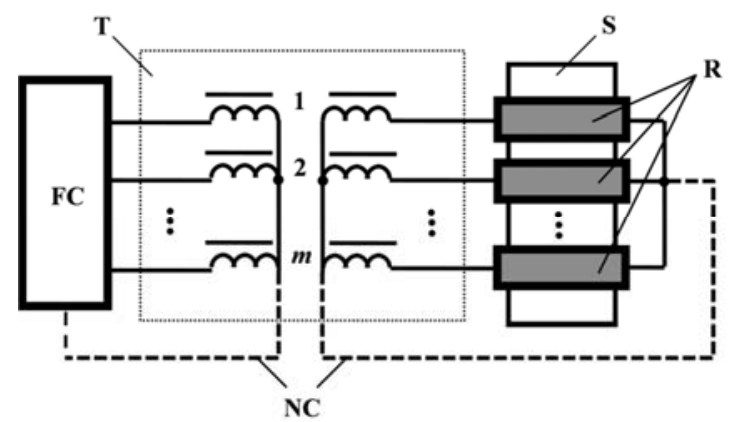

Figure 7. Connection of m-rod stator winding to a m-phase frequency converter according to $B D V-1$, where $F C$ is the frequency converter, $T$ is $m$ phase transformer stepping down voltage, $S$ is stator of an induction motor, $R$ are the rods of stator winding, and NC are neutral conductors.

The application of any one of these design versions allows to achieve the fulfillment of the $H$-invariance law.

Below above mentioned three basic design versions (BDV-1, BDV-2, and BDV-3) are illustrated by concrete examples.

According to the basic design version BDV-1 the AC motor stator winding set consists of the individual rods located in stator slots and insulated from stator magnetic circuit. The rods can be made from copper, aluminum, etc. Every rod is connected to an individual phase of a frequency converter by one of its ends (Figure 5, 6 and 7). Other rods ends are connected in star having or not having a neutral conductor. The rods of the stator winding are connected to the frequency converter via an $m$-phase transformer stepping down voltage. In this case $m$ is the number of the rods (i.e. the number of real phases of the drive system). Such winding design is simpler than the traditional ones, but in this case it is necessary to use transformer stepping down voltage, which has large mass-and-overall dimensions. This design version may be used for both linear and non-linear AC motors.

Such design can be used not only in the case when the motor rotor winding set consists of the individual rods, but also in the case when this winding set is of any usual type (for example, winding set of drum type) [30]. In this case such AC motor design version will allow to decrease the magnetic losses in the motor (Figure 8). 


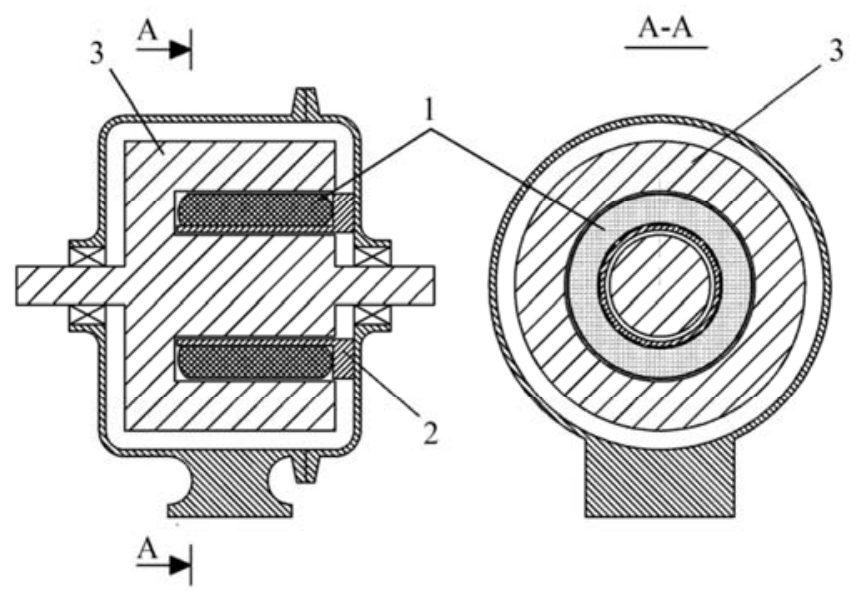

Figure 8. The induction motor having the E-shaped rotor and a stator winding set of any usual type, where 1 is stator winding set of usual type, 2 former of the stator winding set, and 3 is the E-shaped rotor core (magnetic circuit).

The design of multiphase induction motor of rotating type worked out according to the basic design version BDV-2 is shown in section in Figure 9. This motor has toroidal-type stator winding set and $E$-shaped rotor enveloping the motor stator both on the outside and on the inside. The motor stator has two rows of slots: the first row is located on the inner surface of the stator core (the inner stator slots), and the second row is located on the outer surface of it (the outer stator slots). The slots disposition on the stator surface of the 8-phase induction motor developed according to the basic design version BDV-2 is shown in Figure 10. Every stator phase winding drops in radial direction in two slots - in one inner slot and in one outer slot.

Exterior view of the 24-phase induction motor stator, which is developing according to the drawing shown in Figure 6, is shown in Figure 11.

The various versions of the $E$-shaped rotor design of such induction motor can be used [20-22]. For example, this rotor can be laminated [28] (Figure 12) or may have solid ferromagnetic body [27] with circular or $U$-shaped axial-andradial slots (Figure 13) made on the rotor surface according [21] for the decrease of the electrical losses in the rotor circuit.

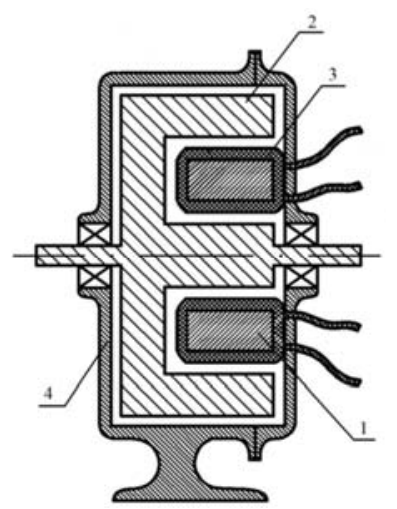

Figure 9. The section of multiphase induction motor of rotating type worked out according to the basic design version $B D V-2$, where 1 is the stator core (i.e. magnetic circuit), 2 is the rotor core (magnetic circuit), and 3 is a stator phase winding, and 4 is the motor case.

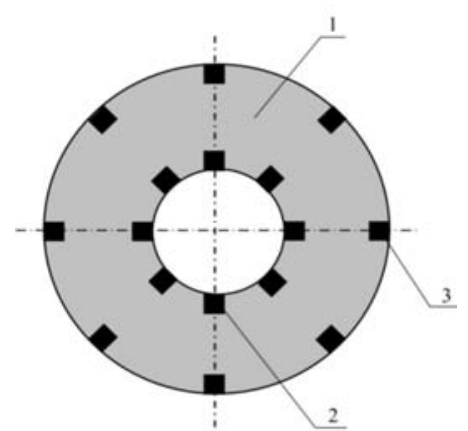

Figure 10. The slots disposition on the stator surface of the 8-phase induction motor developed according to the basic design version $B D V-2$, where 1 is the stator core (i.e. stator magnetic circuit), 2 is the an inner stator slot, and 3 is an outer stator slot.

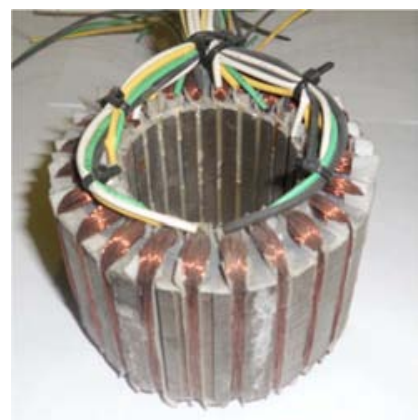

Figure 11. Exterior view of the 24-phase induction motor stator developing according to the drawing shown in Figure 9.

The stator of a 6-phase flat type $\mathrm{AC}$ linear motor, which is worked out according to the basic design version BDV-3 is presented in Figure 14. In this case every stator phase winding drops in two slots separated from each other by diamagnetic shield $D S$. The diamagnetic shield $D S$ divides the magnetic field created by the stator winding into two subfields separated in space. The change of magnetic field pole number on space intervals between borders of everyone above mentioned subfield is observed during PPM application process.

Rotation version of this motor is shown in Figure 15. 


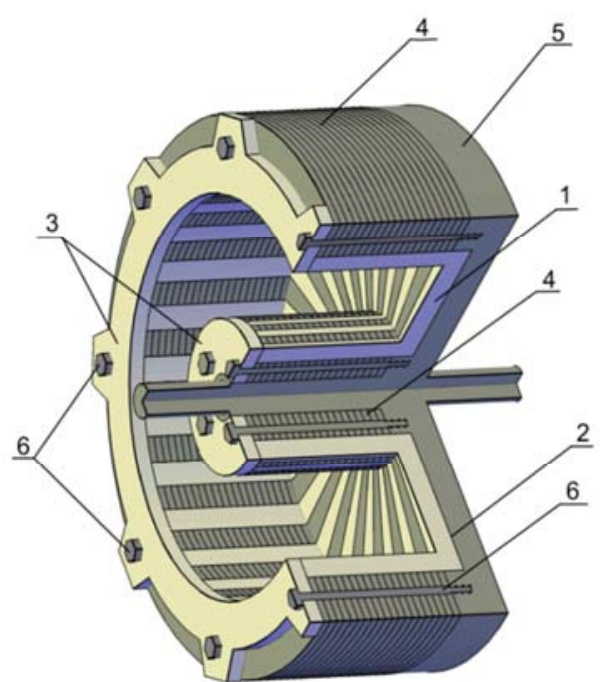

Figure 12. E-shaped laminated rotor, where 1, 2 are U-shaped axial-andradial rods, 3 are short-circuited rings, 4 is laminated part of rotor base, 5 solid part of rotor base, 6 are studs.

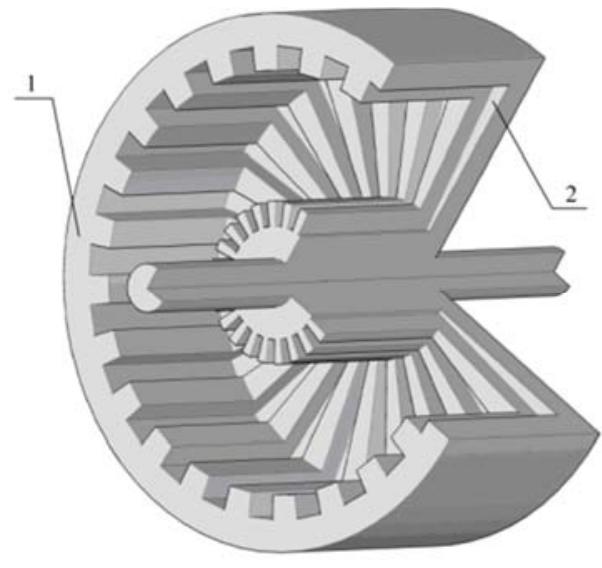

a)

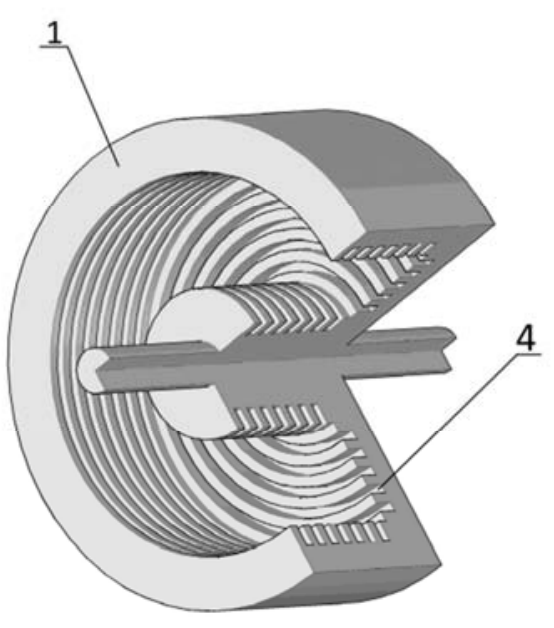

b)

Figure 13. E-shaped solid rotor, where (a - having U-shaped axial-andradial slots; $b$ - having circular slots), where 1 is rotor body, 2 U-shaped axial-and-radial rods, 4 is 3 are short-circuited rings, 4 is laminated part of rotor base, 5 solid part of rotor base, 6 are studs.

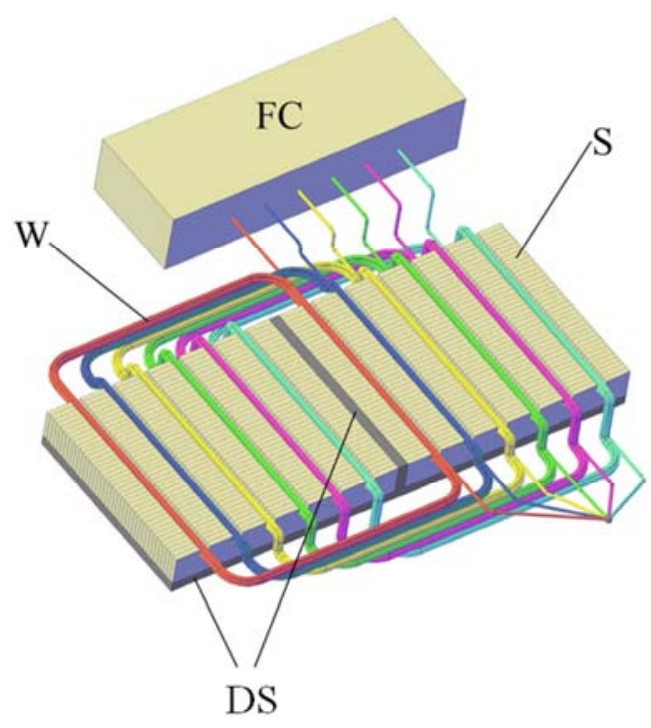

Figure 14. Design version of the stator of a 6-phase flat type AC linear motor and winding connections, where FC is a 6-phase frequency converter, $S$ is the motor stator, $W$ is phase multiple-turn winding of the motor stator, and DS is diamagnetic shield.

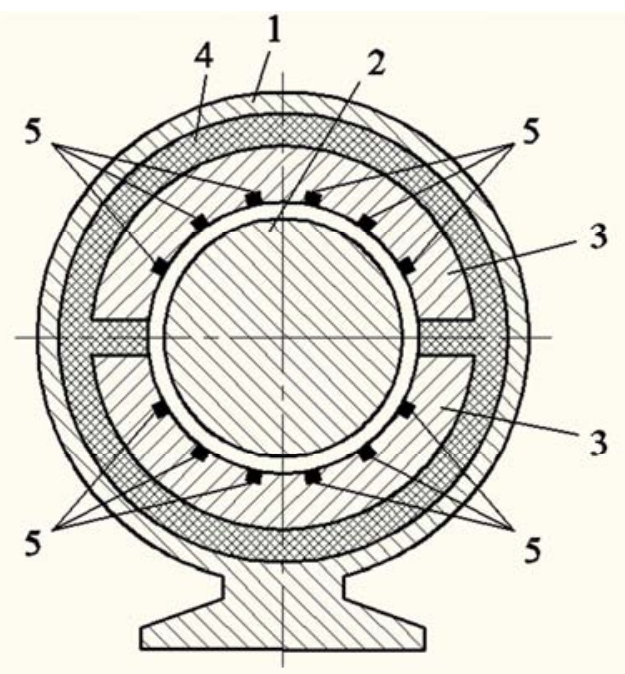

a)

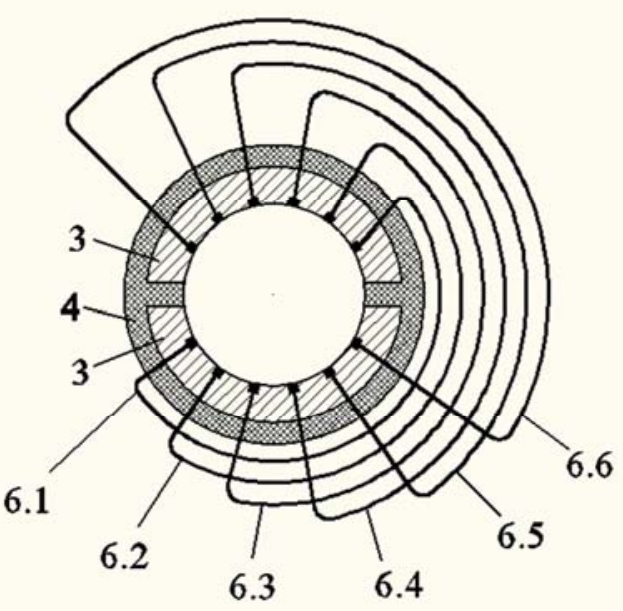

b) 


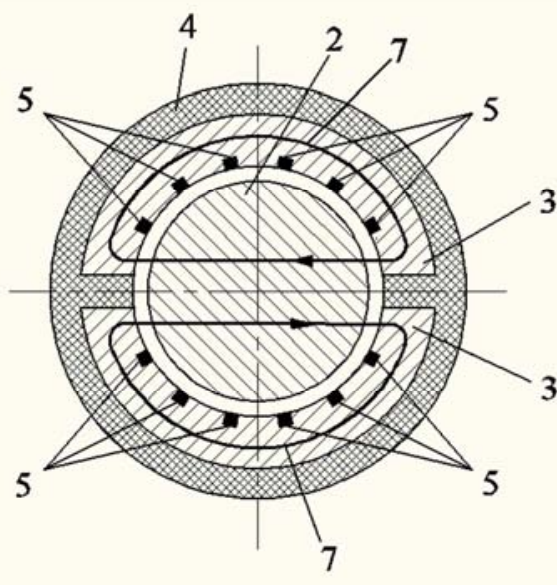

c)

Figure 15. Rotating version of $B D V-\mid 3$, where 1 is the motor stator, 2 is the motor rotor, 3 is the stator core, 4 is diamagnetic shield, 5 are the stator slots, 6.1-6.6 are the stator phase windings, 7 is magnetic field in the motor.

\section{Novel Generation of Hybrid AC Drives}

At present the hybrid traction drives based on the 3-phase induction motors have found a wide application in the field of vehicles of different types (i.e. ground, marine, air vehicles). Among the grave disadvantages of them is a high energy loss in inverter which energizes the 3-phase induction motor (or motors). The existence of these losses is explained by high switching frequency of inverter transistors.

To eliminate this disadvantage it is necessary to use the radically new principles of hybrid AC traction systems designing. According to these novel principles the above mentioned systems must not contain any power electronic elements operating with high frequency.

The creation of such novel hybrid traction systems is possible on the base of multiphase (i.e. having the number $m$ of phases more than four) AC generators (for example, Diesel generators) and non-traditional controlled multiphase induction motors [21-25].

The prospects for the use of multiphase asynchronous linear and non-linear drives in the field of vehicles of different types (i.e. ground, marine, air vehicles) were presented and described in a number of papers (for example, $[17,19])$.

However the increase of the phase number of asynchronous electric drive system allows not only to improve a number of its technical-and-economic characteristics, but also to create the hybrid traction drives according to scheme "AC Diesel-generator - induction motor(s)" which will differ fundamentally from the existing ones, and have more simple design and control unit and less energy losses (in comparison with analogous existing hybrid drives). These hybrid traction drives of novel type are based on the use of the multiphase AC Diesel generators and nontraditional controlled multiphase induction motors.

The basic principles of design of these hybrid traction drives of novel generation are presented in this paper. These basic design principles are the following:

- the increase of AC Diesel generator and induction motor phase numbers more than four;

- the application of such non-traditional methods of control by multiphase induction motor as the oversynchronous control method (OSM) and the phase-pole one (PPM) which are the kinds of the over-phase control method (OPM) [17, 19];

- the use of a number of novel design versions of multiphase AC induction motor.

PPM-controlled multiphase induction motor is in effect a multi-speed motor having only one winding set that is equal (in its complexity) to the winding set of 3-phase single-speed induction motor. The quantity of discrete values of the motor speed rises if the phase number of PPM-controlled multiphase AC induction motor increases.

Because of this there is no need to use a transistor inverter in such multiphase hybrid traction drive to regulate the induction motor rotor speed. The corresponding thyristor or electromechanical commutator is used in the multiphase hybrid traction drive of novel type instead transistor inverter. The operating frequency of such commutator is much less than the operating frequency of above mentioned inverter IGBTs. Owing to this the energy losses are much less and the motor control process is significantly simpler in the multiphase hybrid traction drive of novel generation (in comparison with the analogous existing hybrid drives).

The combined application of OSM and PPM are necessary for obtaining of the following:

- wide range of the motor speed regulation;

- small spacing (step) of the stepwise regulation of the motor speed.

The functional diagram of the power section of the proposed hybrid traction drive system is presented in Figure 16.

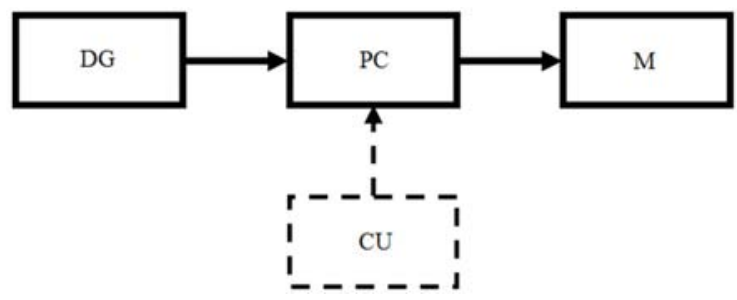

Figure 16. Functional diagram of the power section of the proposed hybrid traction drive system, where DG is m-phase Diesel generator, $P C$ is thyristor or electromechanical phase commutator, $M$ is m-phase induction motor, and $C U$ is control unit of the hybrid traction drive system.

In this hybrid drive system the change of motor speed is reached by the change in the version of connection output terminals of $m$-phase AC generator (for example, Diesel generator) $D G$ to the terminals of the stator phase windings of a $m$-phase induction motor $M$. This change is reached by the phase commutator $P C$ according to the corresponding signals which are derived from the control unit $C U$ of the proposed hybrid traction drive system.

The mode of operation of the multiphase Diesel generator is optimal constantly. 


\subsection{The Design of Diesel Generator}

The number of phases of the AC Diesel generator is more than four $(m>4)$. It is its sole constructive difference from traditional 3-phase AC Diesel generator. The winding set of the $\mathrm{m}$-phase Diesel generator is of traditional drum type. The number $m$ of phases of this winding set must be equal to the phase number of the $\mathrm{AC}$ induction motor.

\subsection{The Commutator}

Phase shifts between of the nearest phase voltages of the motor must be increased by a factor of some whole number when OPM is used. Because of this the transition from traditional control to OPM is possible by the change in order of the connection between the motor phase windings and motor power supply phases (for example, generator phases) by application of the commutator (without any use of an inverter).

The electronic or mechanical phase commutator $P C$ (see Figure 16) must connect output terminals of generator to the terminals of the motor stator phase windings according to the following algorithm:

$$
i_{M}=N_{1}-N_{2},
$$

where

$$
\begin{gathered}
N_{1}=H \cdot\left(i_{i n}-1\right)+1, \\
N_{2}=\left\{\begin{array}{c}
0 \text { if } H=1, \\
m \cdot\left[\left(N_{1}-1\right) / m\right] \text { if } H \geq 2,
\end{array}\right.
\end{gathered}
$$

$i_{M}$ is the number of a motor stator phase winding, $i_{\text {in }}$ is the number of an Diesel generator phase, and $\left[\left(N_{1}-1\right) / m\right]$ is the integer part of the number $\left(N_{1}-1\right) / m$.

The peculiarities of the phase commutator $P C$ may be conveniently shown by the example of the following case (see Figure 17):

- $m=6$,

- 6-phase inverter has traditional scheme and operates at the sole value of the parameter $H: H=1$,

- the change in the parameter $H$ value is achieved by phase commutator $P C$,

- the parameter $H$ may be equal to 1 or 2,

- the logic signal $h$ depends on the value of the OPM parameter $H$ (i.e. $h=1$ if $H=1$, and $h=0$ if $H=2$ ),

- and $T C_{j}$ is the electronic or mechanical terminal changer for the phase having number $j=i+1, i \in[1 ; m], j \in[1 ; m$ $1]$.

If the drive system is low-powered, the type of electronic terminal changers TC may be logic. The electronic logic version of the terminal changer $T C_{j}$ is shown in Figure 18 (for the case of low-power drive system), where the unit " $\&$ " is an AND-type logic gate, and the unit " $\geq 1$ " is an OR-type logic gate. The mechanical switching analogue of the logic scheme shown in Figure 18 is presented in Figure 19.

These schemes carry out the following logic function:

$$
y_{j}=a_{j} \& h \vee b_{j} \& \bar{h}=\left\{\begin{array}{lll}
a_{j} & \text { if } & h=1 \\
b_{j} & \text { if } & h=0 .
\end{array}\right.
$$

\subsection{Possibility to Decrease the Step of Speed Changing}

The main disadvantage of the proposed hybrid drive system is the stepwise change of the motor speed.

However it is possible to obtain some quasi-continuous change of this speed by the decrease in the step of its change. This speed change step decrease can be obtained by the use of the following methods:

Method 1. By the choice of some great value of the parameter $H$ (not $H=1$, but $H \gg>1$ ) as the nominal value of this parameter. For example, the above mentioned speed change step decrease may be characterized by coefficient $V_{S} / V_{S+1}$, where $V_{S}$ is the motor movable secondary element motion speed before the change of the parameter $H, V_{S+1}$ is this speed after the change of the parameter $H$, and $S$ is the step of the parameter $H$ change. The values of the coefficient $V_{S} / V_{S+1}$ are shown in Table 2 for the case when $m=360$ and the parameter $H$ changes from its least value (i.e. $H=1$ ) to the greatest one (i.e. $H=5$ ), where $H_{O P}$ is the value of the parameter $H$ chosen as the nominal value of this parameter.

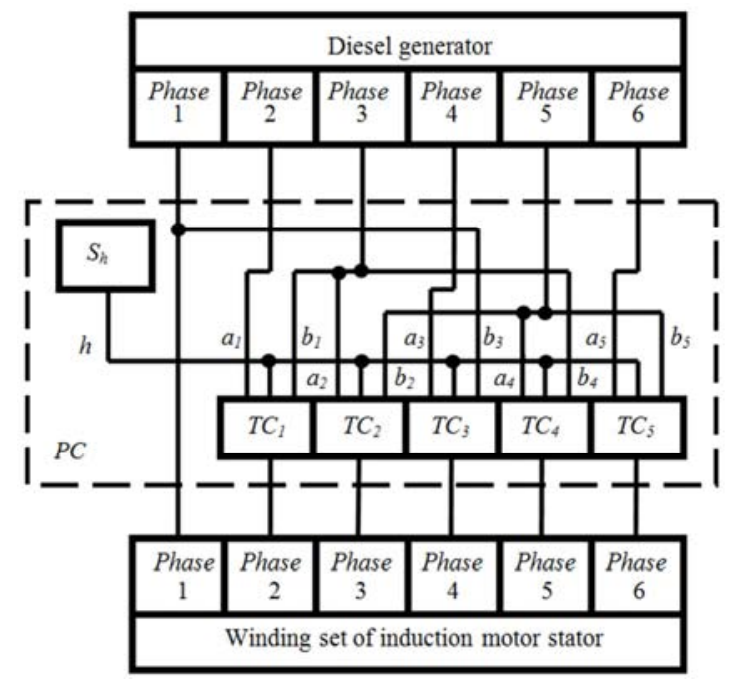

Figure 17. Structural diagram of the 6-phase single-motor OPM-controlled system "Diesel generator - induction motor" with phase commutator between Diesel generator and induction motor, where $S_{h}$ is the source of the signal $h$, and TC are the electronic or mechanical terminal changers.

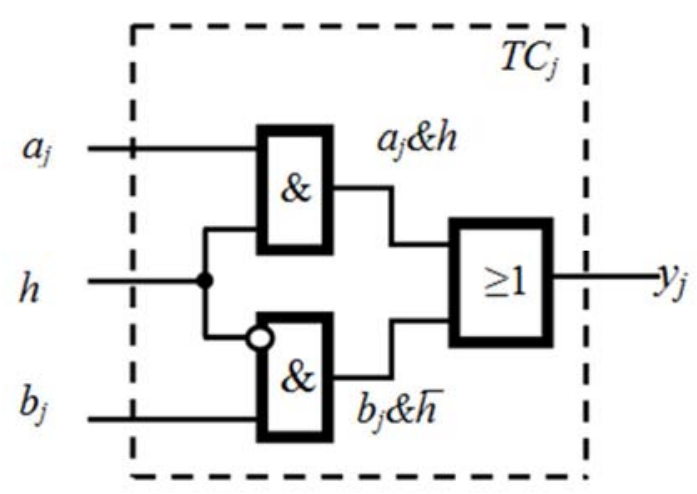

Figure 18. Logic version of the electronic terminal changer $T C_{j}$. 


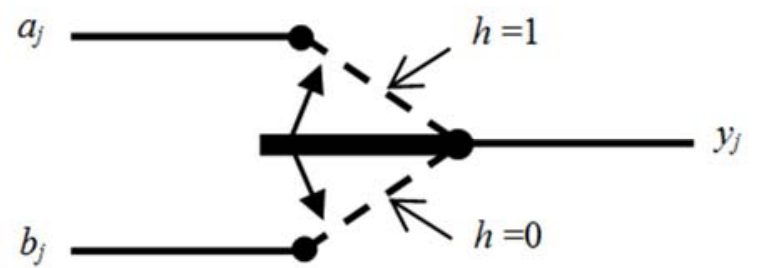

Figure 19. Mechanical switching analogue of the logic scheme shown in Figure 18.

Table 2. The values of the coefficient $V_{S} / V_{S+1}$ for the case when $m=360$ and the parameter $H$ changes from its least value (i.e. $H=1)$ to the greatest one (i.e. $H=5$ ).

\begin{tabular}{lllll}
\hline \multirow{5}{*}{$\boldsymbol{S}$} & $\boldsymbol{H}_{\boldsymbol{O P}}$ & & & \\
\cline { 2 - 5 } & $\mathbf{1}$ & $\mathbf{2}$ & $\mathbf{3}$ & $\mathbf{4}$ \\
\hline 1 & 2,0 & 1,5 & $1,(3)$ & 1,25 \\
2 & 1,5 & $1,(3)$ & 1,25 & - \\
3 & $1,(3)$ & 1,25 & - & - \\
4 & 1,25 & - & - & - \\
\hline
\end{tabular}

Method 2. By the use of not 1-, but 2-stage (2-level) commutator [31] shown in Figure 20. In this case the 1st commutator stage implements the transition to PPM, and the 2nd commutator stage implements the transition to OSM. The combined operation of both commutator stages will allow to decrease the motor speed change step.

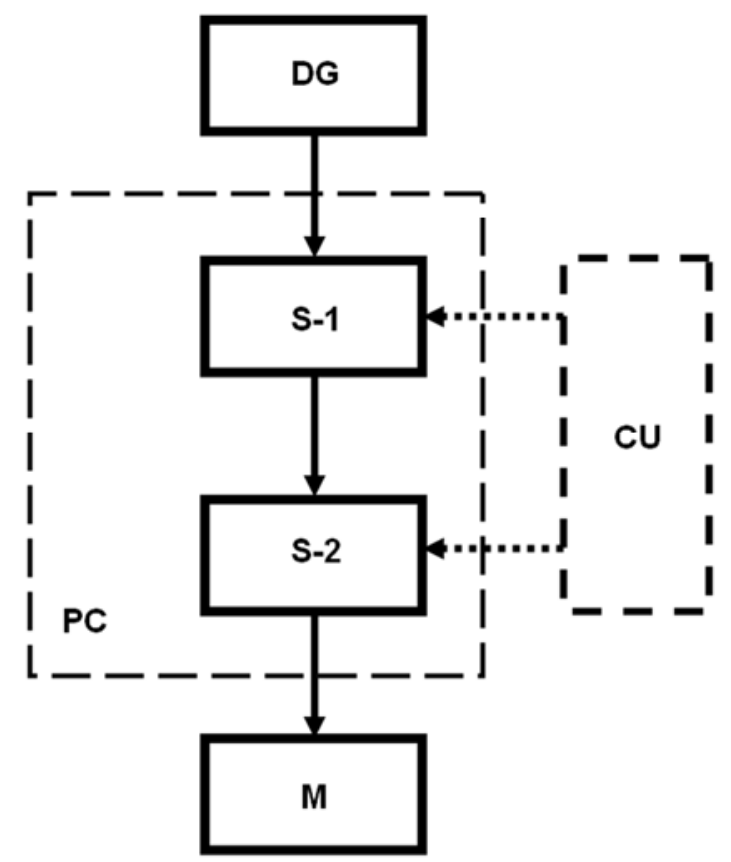

Figure 20. Functional diagram of the power section of the proposed hybrid traction drive system having 2-stage commutator, where $S-1$ is the 1 st stage of $P C$, and $S-2$ is the 2nd stage of $P C$.

Method 3. By the combined use of the above mentioned two methods (the method 1 and method 2).

The increase of the phase number of asynchronous electric drive system allows not only to improve a number of its technical-and-economic characteristics, but also to create the hybrid traction drives according to scheme "Diesel-generator - AC induction motor (s)" without application of any power electronic elements operating with high frequency, which will differ fundamentally from the existing ones, and have more simple design and control unit and less energy losses (in comparison with analogous existing hybrid drives).

\section{Conclusion}

The above-mentioned laws of ACID efficiency invariance are particular cases of the fundamental law (or principle), which prevails in the field of multiphase AC drives and may be stated as follows: for the ensuring of maximal energy efficiency of multiphase ACID the laws of space-temporal spectral relations, which acts in the field of these systems, must be necessarily fulfilling during the process of both ACID structural elements design and motor control mode elaboration.

\section{References}

[1] Brazhnikov, A. V.: Multiphase Inverter Electric Drive with Different Versions of Induction Motor Rotor. Ph. D. dissertation. - Department of Electrical Machines and Drives, Tomsk Polytechnic Institute, Tomsk, Russia, 1985, 210 p. (in Russian).

[2] Brazhnikov, A. V.; Kochetkov, V. P.: Multiphase Frequency Controlled Inverter Electric Drive with Induction Motor. Collection of papers presented at 6th International Conference on Electrical Machines and Drives ELMA '90. - Varna, Bulgaria, October 1990, paper No 12, 10 p. (in Russian).

[3] Brazhnikov, A. V.; Kochetkov, V. P.: Multiphase Frequency Controlled Inverter Electric Drive with Induction Motor. Proc. of 6th International Conference on Electrical Machines and Drives ELMA '90. - Varna, Bulgaria, October 1990, p. 44 (in Russian).

[4] Brazhnikov, A. V.: Additional Resources of Control of Multiphase Inverter Drives. Proc. of 7th International Conference on Electrical Machines and Drives ELMA '93. Varna, Bulgaria, October 1993, pp. 325-332.

[5] Brazhnikov, A. V.; Dovzhenko, N. N.: Beyond Routine Control of Multiphase Inverter Drives. Proc. of International Conference on Power Electronics, Motion Control and Association Applications PEMC '94, vol. 1. - Warsaw, Poland, September 1994, pp. 99-104.

[6] Brazhnikov, A. V.; Dovzhenko, N. N.; Izmaylov, E. B.: Prospects for the Use of Multiphase Electric Drives in Field of Mining Machines. Proc. of 3rd International Symposium on Mine Mechanization and Automation MMA '95, vol. 1. Golden, Colorado, USA, June 1995, pp. 13-13 - 13-23.

[7] Brazhnikov, A. V.; Dovzhenko, N. N.; Gilyov, A. V.; Butkin, $\mathrm{V}$. D.: Improvement of Technical-and-Economic Characteristics of Drilling Rigs Owing to the Use of Multiphase Electric Drives. Proc. of ISDT 16th Annual Technical Conference. - Las Vegas, Nevada, USA, May 1996, 8 p.

[8] Brazhnikov, A. V.; Dovzhenko, N. N.: Advantages of Multiphase Electric Drives - Application in Drilling Rigs. Proc. of 4th International Symposium on Mine Mechanization and Automation MMA '97, vol. 1. - Brisbane, Queensland, Australia, July 1997, pp. B4-37 - B4-42. 
[9] Brazhnikov, A. V.; Dovzhenko, N. N.: Control Potentials and Advantages of Multiphase AC Drives. Proc. of 29th Annual IEEE Power Electronics Specialists Conference PESC '98. Fukuoka, Japan, May 1998, vol. 2, pp. 2108-2114.

[10] Brazhnikov, A. V.; Belozyorov, I. R.: Over-Phase Control of Inverter Multiphase AC Linear Drives. Proc. of 8th International Symposium on Linear Drives for Industry Application LDIA '2011. - Eindhoven, Netherlands, July 2011, paper No 172, 6 p., CD-ROM.

[11] Brazhnikov, A. V.; Belozyorov, I. R.: Over-Phase Control of Inverter Multiphase AC Linear Drives. Book of Abstracts of 8th International Symposium on Linear Drives for Industry Application LDIA '2011. - Eindhoven, Netherlands, July 2011, pp. 122-123.

[12] Brazhnikov, A. V.; Belozyorov, I. R.; Molokitin, S. A.: Inverter-Fed Multiphase AC Linear Motors under NonTraditional Control. Proc. of 21st International Conference on Magnetically Levitated Systems and Linear Drives MAGLEV '2011. - Daejeon, South Korea, October 2011, paper No PLE04, 5 p., CD-ROM.

[13] Brazhnikov, A. V.; Belozyorov, I. R.; Molokitin, S. A.: Energy Efficiency Invariance Laws Acting in the Field of Multiphase Inverter-Fed AC Linear Motors. Proc. of 21st International Conference on Magnetically Levitated Systems and Linear Drives MAGLEV '2011. - Daejeon, South Korea, October 2011, paper No PLE-06, 4 p., CD-ROM.

[14] Brazhnikov, A. V.; Belozerov, I. R.: Non-Traditional Control and Advantages of Multiphase AC Inverter Drives. Proc. of IEEE International Conference on Energy, Automation and Signal ICEAS '2011. - Bhubaneswar, Orissa, India, December 2011, pp. 781-786.

[15] Brazhnikov, A. V.; Belozerov, I. R.: Energy Efficiency Invariance Laws Acting in the Field of Multiphase AC Inverter Drives. Proc. of IEEE International Conference on Energy, Automation and Signal ICEAS '2011. Bhubaneswar, Orissa, India, December 2011, pp. 787-792.

[16] Brazhnikov, A. V.; Belozerov, I. R.: Space-Temporal Spectral Relations and Energy Efficiency Invariance Laws Acting in the Field of Inverter-Fed Multiphase AC Drives. Proc. of IET 6th International Conference on Power Electronics, Machines and Drives PEMD '2012. - Bristol, UK, March 2012, vol. 2, pp. 1094-1099.

[17] Brazhnikov, A. V.; Belozerov, I. R.: Over-Phase Control of Inverter Multiphase AC Linear Drives. Journal "Mechatronics". - Elsevier Publishing Company, vol. 23, issue 2, March 2013, pp. 227-232.

[18] Brazhnikov, A. V.; Belozerov, I. R.: Prospects for the Use of Multiphase Inverter-Fed Asynchronous Drives in the Field of Traction Systems of Railway Vehicles. Journal "International Journal of Railway". - South Korea, vol. 5, No 1, March 2012, pp. 38-47.

[19] Brazhnikov, A. V.; Brazhnikova, E. S.; Belozerov, I. R.: PPMBased Development-and-Control Strategy of Fault Tolerant Inverter-Fed Multiphase Electromechanical AC Systems. Proc. of 21st International Symposium on Power Electronics,
Electrical Drives, Automation and Motion SPEEDAM '2012. - Sorrento, Italy, June 2012, pp. 237-242.

[20] Brazhnikov, A. V.; Brazhnikova, E. S.: Efficiency Invariance Laws and Development of Multiphase AC Inverter Drives. Proc. of 21st International Symposium on Power Electronics, Electrical Drives, Automation and Motion SPEEDAM '2012. - Sorrento, Italy, June 2012, pp. 420-425.

[21] Brazhnikov, A. V.; Brazhnikova, E. S.: Novel Generation of Hybrid Traction Drives With AC Induction Motors for Railway Vehicles - Principles of Designing. Proc. of 2012 International Session at Annual Conference \& Meeting of the Korean Society for Railway ISKSR '2012, GyeongJu, South Korea, October 2012, pp. 44-49.

[22] Brazhnikov, A.; Dovzhenko, N.; Minkin, A.; Pomolotova, O.; Litvinenko, A.; Shilova, V.: Peculiarities of Novel Generation of Hybrid Traction Systems for Electrical Vehicles. Advanced Science and Technology Letters, vol. 37 (Electrical Engineering 2013), pp.17-20. - JeJu Island, South Korea.

[23] Brazhnikov, A.; Dovzhenko, N.; Minkin, A.; Pomolotova, O.; Litvinenko, A.; Shilova, V.: Novel Type of EV Hybrid Traction Drives. International Journal of Control and Automation. South Korea, vol. 7, No 3, March 2014, pp. 251-266.

[24] Brazhnikov, A. V.; Dovzhenko, N. N.; Minkin, A. N.; Pomolotova, O. V.; Litvinenko, A. I.; Shilova V. A.: Multiphase Hybrid Traction Drives for Electrical Vehicles. Proc. of the 22nd International Symposium on Power Electronics, Electrical Drives, Automation and Motion SPEEDAM '2014. - Ischia Island, Naples Bay, Italy, June 1820, 2014, pp. 583-588.

[25] Brazhnikov, A.; Dovzhenko, N.; Minkin, A., Loveiko, M.: Extraordinary Properties and Some Design Peculiarities of OPM-Controlled Multiphase Asynchronous Drives. Journal "Open Science Journal of Electrical and Electronic Engineering”. - USA, vol. 2, No. 5, 2015, pp. 78-83.

[26] Brazhnikov, A. V.; Belozerov, I. R.: Inverter Multiphase Induction Motor Drive under Phase-Pole Control. Russian patent No RU 100863 U1 dated 27.12.2010 (in Russian).

[27] Brazhnikov, A. V.; Belozerov, I. R.; Molokitin, S. A.: Solid Ferromagnetic Rotor for Phase-Pole-Controlled Induction Motor. Russian Patent No RU 109345 U1 dated 10.10.2011 (in Russian).

[28] Brazhnikov, A. V.; Belozerov, I. R.: E-Shaped Rotor Having Short-Circuited Winding for Phase-Pole-Controlled Induction Motor. Russian patent No RU 118805 U1 dated 27.07.2012 (in Russian).

[29] Brazhnikov, A. V.; Belozerov, I. R.: Induction Motor Having E-Shaped Rotor. Russian patent No RU 116720 U1 dated 27.05.2012 (in Russian).

[30] Brazhnikov, A. V.: Multiphase Induction Motor. Russian patent No RU 127268 U1 dated 20.04.2013 (in Russian).

[31] Brazhnikov, A. V.; Brazhnikova, E. S.; Bondarenko, R. V.; Urchukov, A. A.: Speed Stepwise Controlled Multiphase Induction Motor Drive. Russian patent No RU 130165 U1 dated 10.07.2013 (in Russian). 